Artículo

\title{
Percepción de citricultores ante el efecto del cambio climático en Campeche
}

\author{
Verónica Rosales Martínez ${ }^{1 \S}$ \\ Alexander Francisco Rubio ${ }^{2}$ \\ Lorena Casanova Pérez ${ }^{3}$ \\ Silvia Fraire Cordero ${ }^{1}$ \\ Carolina Flota Bañuelos ${ }^{1}$ \\ Francisco Galicia Galicia ${ }^{4}$
}

${ }^{1}$ Cátedras CONACYT. Colegio de Postgraduados-Campus Campeche. Carretera Haltunchén-Edzná km 17.5, Sihochac, Champotón, Campeche, México. CP. 24450. (frairec@colpos.mx; cflota@colpos.mx). ${ }^{2}$ Instituto Tecnológico de Chicontepec. Barrio dos Caminos Núm. 22, Chicontepec, Veracruz. (alex22fco@gmail.com). ${ }^{3}$ Universidad Tecnológica de la Huasteca Hidalguense. Carretera HuejutlaChalahuiyapa km 3.5, Col. Tepoxteco, Hidalgo. CP. 43000. (lorena.casanova@uthh.edu.mx). ${ }^{4}$ Investigador Independiente. Agua Santa, Comapa, Veracruz. CP. 94210. (frgalicia@hotmail.com).

${ }^{\S}$ Autora para correspondencia: vrosales@colpos.mx.

\section{Resumen}

En México, el cultivo de cítricos tiene una importancia alimentaria, social y cultural que llega a regir la vida familiar y la dinámica comunitaria en las regiones y están siendo afectadas por el cambio climático. El objetivo de esta investigación fue medir la percepción de los citricultores, ante el efecto del cambio climático. Se aplicó una encuesta participativa a 65 productores de cítricos de Campeche, México. Se midieron variables sobre ocurrencia de eventos asociados al cambio climático, daño directo a las plantas, percepción del aumento de fenómenos meteorológicos, cambio tecnológico y socioeconómico. Los encuestados son hombres $(86.3 \%)$, de edad mayor y escolaridad básica. El 55\% de los participantes posee tierra ejidal y $44 \%$ propiedad privada. El rendimiento promedio de los cítricos es bajo debido a la falta de sistemas de riego tecnificado y a los efectos del cambio climático, particularmente el aumento de la temperatura y el descenso de la precipitación. Los productores poseen un total de siete tipos de cultivos en sus parcelas, sin correlación con el tamaño de propiedad $(r=0.032)$, ni con el área destinada al cultivo $(r=0.33)$. El $66.6 \%$ de los participantes ha escuchado sobre el cambio climático siendo la fuente de información la televisión. El 69.6\% ha percibido cambios mayores en el clima, lo que ha motivado a realizar cambios en el tipo de labranza (McNemar.test $\chi^{2}=10.56, p<0.01^{* *}$ ). Así, los efectos del cambio climático están incidiendo en las prácticas de manejo de los cítricos en Campeche; sin embargo, son acciones reactivas y se requieren de investigaciones que cuantifiquen y valoren los daños por este fenómeno y se diseñen estrategias para contrarrestar sus efectos en el mediano y largo plazo.

Palabras clave: cambios, citricultura, clima, productores, vulnerabilidad.

Recibido: enero de 2020

Aceptado: marzo de 2020 


\section{Introducción}

La citricultura es una actividad que se desarrolla en climas tropicales y subtropicales. A nivel nacional, Michoacán ocupó el primer lugar en producción de limón, con 711181 t y un rendimiento de $16.047 \mathrm{t} \mathrm{ha}^{-1}$, seguido por Veracruz con $661733 \mathrm{t}$. Las zonas productoras de cítricos en México están bien delimitadas, en la costa del Golfo de México, se cultivan en estados como Tamaulipas, Veracruz, Tabasco y Yucatán, y de menor relevancia en Nuevo León y Puebla. Otra zona es la de la costa del Pacífico en estados como Sonora, Colima, Michoacán y Oaxaca.

La naranja es el cítrico con mayor cantidad de hectáreas sembradas y cosechadas en México (58.9\%) (60.5\%), le siguen el limón (34\%) (32.2\%); la mandarina (3.8\%) (4\%) y la toronja (3.4\%) (3.3\%) (SIAP, 2019). Esto contribuye a que México se encuentre en una posición importante a nivel mundial con respecto a la producción de cítricos: $5^{\text {to }}$ lugar como productor de naranja, $2^{\text {do }}$ lugar como productor de limón, $4^{\text {to }}$ lugar como productor de toronja y $13^{\text {vo }}$ lugar como productor de mandarina (SAGARPA, 2018).

El limón mexicano (Citrus aurantifolia) ha encontrado en el mercado japonés su principal comprador, se exporta anualmente alrededor de dos mil toneladas (Maya, 2017). Por su parte, el estado de Campeche reportó una superficie sembrada de limón de 2018 ha, considerando el total del cultivo, con una producción de $13453 \mathrm{t}$ y un rendimiento de $7.539 \mathrm{t} \mathrm{ha}^{-1}$ (SIAP, 2017). No obstante, las condiciones tropicales y las precipitaciones, son los principales factores climáticos que influyen sobre el crecimiento, desarrollo y productividad de las plantas de cítricos (OrduzRodríguez et al., 2017; Agovino et al., 2018).

La permanencia de dificultades climáticas en el sector citrícola tiene principal efecto negativo en la baja productividad de los huertos y por ende en el abandono gradual de las plantaciones. Este fenómeno de cambios en las magnitudes y distribuciones meteorológicas de las variables climáticas clásicas que incluyen la temperatura, precipitación, humedad, velocidad del viento y la evaporación, provocarán cambios en los comportamientos fisiológicos de las plantas y a su vez impactarán en la productividad agrícola a escala global (Orduz-Rodríguez et al., 2017; Zhang et al., 2017; Agovino et al., 2018).

De esta manera, el cambio climático plantea serios desafíos para la agricultura y la adaptación a sus impactos (Klocker et al., 2018) por lo que los productores y sus sistemas de producción son cada vez más vulnerables a sus efectos. El Panel Intergubernamental sobre Cambio Climático (IPCC) define vulnerabilidad ante el cambio climático como el 'grado de susceptibilidad o de incapacidad de un sistema para afrontar los efectos adversos del cambio climático y en particular, la variabilidad del clima y los fenómenos extremos. La vulnerabilidad dependerá del carácter, magnitud y rapidez del cambio climático a que esté expuesto un sistema, y de su sensibilidad y capacidad de adaptación' (Ibarrarán et al., 2014).

En el debate actual sobre el cambio climático, los temas de vulnerabilidad social y percepciones de la población frente a dicho fenómeno ocupan un lugar destacado, principalmente porque nutre el conocimiento sobre los intereses, demandas y necesidades de los diferentes sectores sociales y permite sentar las bases para construir procesos de participación social orientados a reducir los riesgos de desastres ocasionados por el incremento de los fenómenos hidrometeorológicos (Sandoval et al., 2014). 
El sector agrícola es sensible al cambio climático y a la capacidad de los pequeños agricultores para desarrollarse (Makuvaro et al., 2018). Por tanto, es necesario adaptar las prácticas actuales y desarrollar nuevas estrategias de resistencia al clima en los sistemas de cultivos (Makate et al., 2019). Otro reto importante de los estudios relacionados con la vulnerabilidad y adaptación al cambio climático es que, a pesar de la existencia de estudios físicos y geográficos sobre impactos de este fenómeno, y a la fecha han permitido establecer escenarios de impactos a nivel territorial.

En este momento no se cuenta con suficiente información a escala reducida sobre la percepción acerca de su vulnerabilidad, o los impactos del fenómeno en el bienestar económico y social de la población (Sandoval et al., 2014), esto puede reducir la eficiencia y eficacia de la implementación de las prácticas de conservación agrícolas, alterar la hidrología, alterar ecosistemas, provocar erosión y afectar la capacidad de los agricultores para desarrollarse (Wagena e Easton, 2018) disminuyendo las ganancias por agricultura (Dussel-Peters, 2002; Pérez et al., 2006).

Aumentando la volatilidad de los precios de los alimentos (López-Feldman y Hernández-Cortés, 2016) y convirtiéndose en una amenaza potencial para la seguridad y soberanía alimentaria de los países en desarrollo, en formas complejas (Kusangaya et al., 2014; Golcher et al., 2017). En la adaptación ante el cambio climático, los esfuerzos gubernamentales se han distinguido por la búsqueda para mejorar la productividad agrícola y el desarrollo del riego basado en la tecnología actual (Islam y Nursey-Bray, 2017; Karimi et al., 2018).

Algunas estrategias implementadas para reorientar el desarrollo agrícola bajo la realidad del cambio climático es la agricultura climáticamente inteligente, la agricultura de conservación, variedades de especies mejoradas entre muchas otras (Islam and Nursey-Bray, 2017; Thornton et al., 2018). En consecuencia, el presente manuscrito pretende mostrar las percepciones de los productores del estado de Campeche, México.

Ante el impacto del cambio climático en el cultivo de cítricos y las estrategias agroecológicas de adaptación ante este fenómeno. Por lo tanto, la hipótesis que deriva de este trabajo es que los productores de cítricos perciben los daños que puede ocasionar el $\mathrm{CC}$, pero aún no establecen estrategias agroecológicas adecuadas para contrarrestar dichos efectos.

\section{Materiales y métodos}

\section{Área de estudio}

La investigación se realizó en dos municipios del estado de Campeche: Champotón y Campeche, debido a que estos municipios están considerados entre los siete que concentran a la mayoría de los productores de cítricos, junto a Escárcega, Hopelchén, Tenabo, Hecelchakán y Calkiní (Tribuna, 2020), Campeche se encuentra en el sureste de la República Mexicana y limita al norte con el estado de Yucatán, al sur con Guatemala y Tabasco, al este con el estado de Quintana Roo y al oeste con el Golfo de México (INEGI, 2012).

Es uno de los estados de la República Mexicana con mayor vulnerabilidad a los efectos del cambio climático. La elevación del nivel medio del mar representa para su franja litoral uno de los retos más apremiantes por la afectación y aceleración de los procesos de erosión en sus playas. Situación que pone en riesgo miles de millones de pesos en infraestructura vital de comunicaciones y transporte, además de afectar las vías terrestres, de gran importancia al ser la entrada a la Península de Yucatán (Ecosur, 2012). 
Campeche es altamente vulnerable a los efectos del cambio climático debido a su ubicación geográfica y su condición de costa, así como por los niveles de marginación y pobreza de su población y la concentración de más de la mitad de sus habitantes en su zona costera (Márquez, 2016). En este estado predomina el clima cálido subhúmedo, que se presenta en $92 \%$ de su territorio, $7.75 \%$ presenta clima cálido húmedo localizado en la parte este del Estado, y en la parte norte, un pequeño porcentaje de $0.05 \%$ con clima semiseco.

La temperatura media anual oscila entre los 26 y $27^{\circ} \mathrm{C}$. La temperatura máxima supera los $30^{\circ} \mathrm{C}$ y la mínima es de $18{ }^{\circ} \mathrm{C}$. Las lluvias son de abundantes a muy abundantes durante el verano y la precipitación total anual varía entre 1200 y $2000 \mathrm{~mm}$, aunque en la región norte, de clima semiseco, es menor, alrededor de $800 \mathrm{~mm}$ anuales. No obstante, las predicciones apuntan a una evolución al alza de temperatura media anual, con un incremento comprendido entre $2.5^{\circ} \mathrm{C}$ y $4{ }^{\circ} \mathrm{C}$ entre 1961 y 2099, debido al aumento previsto en las temperaturas mínimas y máximas (gobierno del estado de Campeche, 2015).

\section{Determinación del tamaño de muestra}

Se calculó del tamaño de muestra utilizando la fórmula (1) propuesta Sierra (1995) para poblaciones finitas, tomando $\mathrm{N}$ de una lista de productores proporcionada por el Comité de Sanidad Vegetal de los municipios de Champotón y Campeche correspondiente a 605 productores en total. Esta ecuación arrojó a 65.21 productores, por lo que se encuestó a 66 productores de cítricos en total.

$$
\mathrm{n}=\frac{\mathrm{N} \times \mathrm{Z}_{\mathrm{a}}^{2} \times \mathrm{p} \times \mathrm{q}}{\mathrm{d}^{2}(\mathrm{~N}-1)+\mathrm{Z}_{\mathrm{a}}^{2} \times \mathrm{p} \times \mathrm{q}}
$$

Donde: $\mathrm{N}=$ tamaño de la población $=604$ productores; $\mathrm{Z}_{\mathrm{a}}=$ nivel de confianza $=1.96^{2}$ ( $\mathrm{si}$ la seguridad es al 95\%); $\mathrm{p}=$ proporción esperada $=5 \%=0.05 ; \mathrm{q}=$ probabilidad de fracaso $=1-\mathrm{p}$ (en este caso $1-0.05)=0.95 ; \mathrm{d}=$ precisión $=5 \%=0.05$.

\section{Recopilación de datos y encuesta}

Se utilizó la encuesta participativa como un diagnóstico para medir la percepción; aplicando cuestionarios a 66 productores de cítricos bajo precepto de colaboración, la única condicionante para aplicar el cuestionario fue que tuvieran cultivo de cítricos sin importar el tipo de cultivo ni la especie de ellos dentro de sus unidades de producción. El cuestionario aplicado consistió en preguntas abiertas y cerradas donde se evaluaron variables de tipo cualitativas y cuantitativas.

Se implementó el muestreo no probabilístico 'bola de nieve' (Hernández-Sampieri et al., 1991). Se emplearon cinco indicadores, los cuales fueron: fenómenos provocados por el CC (Cambio Climático) que afectan a la citricultura, efectos del CC en la citricultura, manejo cultural ante el efecto del $\mathrm{CC}$, percepción del nivel de aumento en situaciones climáticas y estrategias agroecológicas ante el efecto del CC. De estos indicadores se obtuvieron variables sobre la ocurrencia de eventos; variables sobre el daño directo a las partes de la planta. 
Como pérdida de ramas, hojas, flor y frutos, erosión y pérdida de nutrientes del suelo, así como disminución en la producción y productividad; todas estas variables fueron medidas a través de un SI, NO. Antes de iniciar con la encuesta, se platicaba con los productores con el fin de valorar el grado de conocimiento que tenían sobre el CC, aunque con sus respuestas no definían con claridad la palabra $\mathrm{CC}$, se percibió que sí tenían el conocimiento sobre lo que este fenómeno implicaba, por lo que se procedió a aplicar el cuestionario.

Además, se evaluaron otras variables como percepción del aumento de sequías prolongadas, lluvias fuertes, huracanes, tormentas, inundaciones y vientos fuertes. Así mismo variables, para medir el cambio en las prácticas culturales ante los efectos del cambio climático anteriormente descritos, como chapeo, barbecho, rastreo, control de maleza, fertilización y abonado, control de plagas y enfermedades, riego y poda, estas variables también se midieron con un si o no.

Para complementar dicha información y establecer relaciones entre variables se midieron cinco variables para reflejar las características socioeconómicas, como la edad del productor, superficie destinada al cultivo de cítricos, escolaridad y género. Así mismo, se realizaron recorridos exploratorios y entrevistas a profundidad con algunos productores de cítricos para conocer con mayor detalle la producción de cítricos y la vulnerabilidad ante los efectos del cambio climático.

\section{Análisis de la información}

Los datos arrojados por el instrumento de diagnóstico fueron analizados con el paquete estadístico $\mathrm{R}$ (3.4.2, 2017). Se realizaron análisis descriptivos para la mayoría de las variables. Las variables para el análisis de componentes principales se convirtieron a valores binarios. Se realizaron correlaciones lineales, en busca de factores relevantes. También se utilizó la prueba no paramétrica de McNemar para detectar cambios en el uso de tecnologías ante cambios ambientales.

\section{Resultados y discusiones}

\section{Características socioeconómicas y productivas de los citricultores}

En el largo plazo puede haber efectos profundos sobre los ecosistemas y la sociedad, y una parte de los efectos que incidan en la población dependerá de la intensidad y frecuencia de los eventos climáticos, así como de la forma en que una comunidad determinada esté lista para enfrentarlos. Por lo que, ante desastres similares, se espera que mientras menos preparada esté la población, mayores serán los efectos negativos.

Esta incapacidad de respuesta o vulnerabilidad está íntimamente ligada con algunas de las características socioeconómicas intrínsecas de la comunidad, que reflejan el nivel de desarrollo y de bienestar en el que vive su población (IPCC, 2007). Por tanto, en esta investigación, la característica socioeconómica 'edad media' de las personas que se dedican al cultivo de cítricos en el estado de Campeche es de 56.5 años $(\mathrm{sd}=14.6, \mathrm{n}=65)$.

Esto quiere decir que son productores de edad avanzada, dato similar al reportado para la mayoría de las personas que se dedican a actividades agrícolas en México (Bahena-Delgado y TorneroCampante, 2009), donde los más jóvenes prefieren dedicarse a otras actividades y han abandonado 
las actividades agrícolas de sus comunidades migrando hacia otras partes del país o hacia los Estados Unidos de América (Rosales et al., 2015). Por su parte, en la distribución de citricultores con respecto al género, los resultados indican que $86.3 \%$ de los productores son del sexo masculino.

Esta tendencia es similar no sólo para agricultores y campesinos dedicados a la citricultura sino también en general para la gente dedicada a alguna actividad del campo, y tiene que ver con la idiosincrasia y cultura del campesinado mexicano (Camargo-López y Espericueta-Reyna, 2006), debido a que por tradición, ha sido el hombre de familia quien se ha dedicado a las labores propias de la agricultura y a la mujer se le ha relegado a las labores propias del hogar (Rosales-Martínez y Leyva-Trinidad, 2019).

El nivel de estudios predominantemente alcanzado entre la población de citricultores es la educación básica $50 \%$ educación primaria y $22 \%$ secundaria y como contraste, solo $4.2 \%$ tienen estudios de licenciatura (Figura 1). Este dato es similar al reportado por Galindo et al. (2000) para 11 regiones establecidas en distintos distritos de desarrollo en zonas campesinas en Zacatecas México y los reportados por Bahena-Delgado y Tornero-Campante (2009).

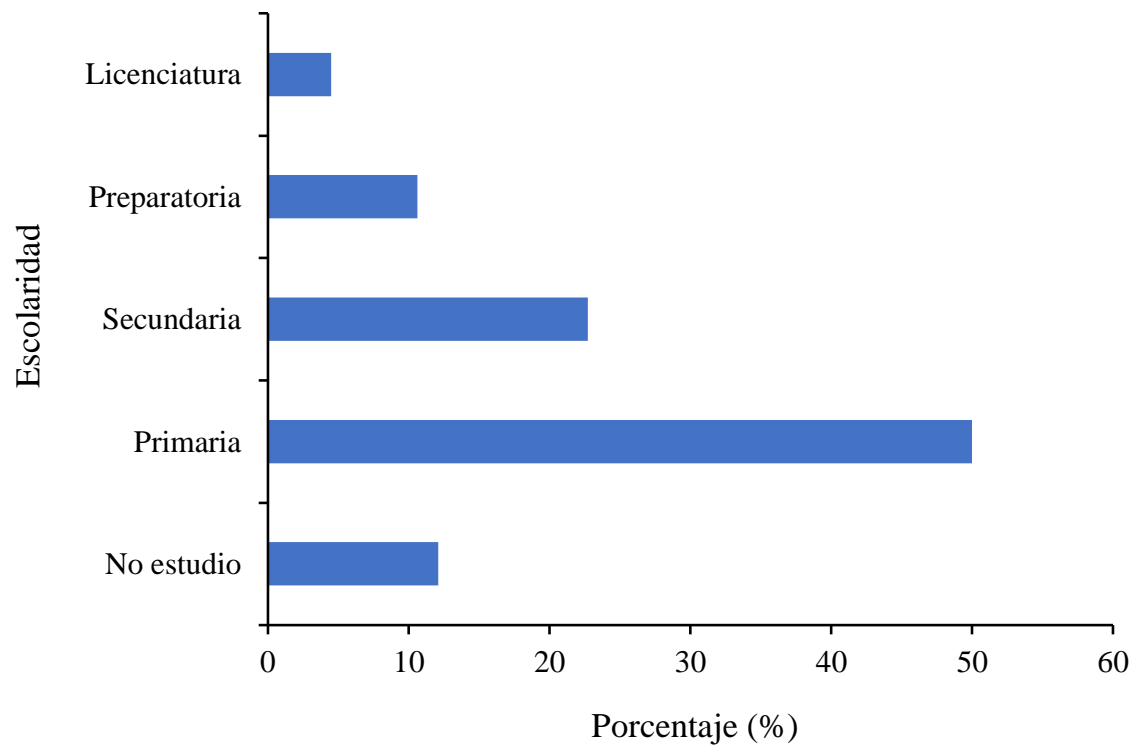

Figura 1. Distribución por escolaridad de los productores de cítricos del estado de Campeche.

Para pequeñas unidades de producción, donde la mayor cantidad del grueso de la población sólo cuenta con educación básica. Corona (2018) menciona que, bajo este contexto cultural, el nivel educativo, así como el género y la ocupación, conforman la percepción individual y grupal sobre el cambio climático. De acuerdo con la tenencia de la tierra, el 55.3\% son parcelas de ejidos y el restante $44.6 \%$ son de propiedad privada.

La tendencia de la tenencia de la tierra es un aproximado de la media nacional que en épocas pasadas era dominada por tierras ejidales (Morett-Sánchez y Cosío-Ruiz, 2017), que vieron disminuida su extensión debido a las reformas agrarias del país, siendo más evidentes durante el tiempo en el Censo Agrícola, Ganadero y Forestal de 2007. El rendimiento por hectárea del cultivo de limón, con variedades de limón dulce y agrio, de acuerdo con el volumen de 
producción en los municipios de Champotón y Campeche (3.03 t productor $\left.{ }^{-1}, \mathrm{sd}=3.54, \mathrm{n}=36\right)$, de la naranja dulce $\left(2.5\right.$ t $\left.^{\text {productor }}{ }^{-1}, \mathrm{sd}=5.06, \mathrm{n}=36\right)$ y de la mandarina $\left(2.34\right.$ t productor $^{-1}$, $\mathrm{sd}=10.03, \mathrm{n}=36)($ Figura 2).

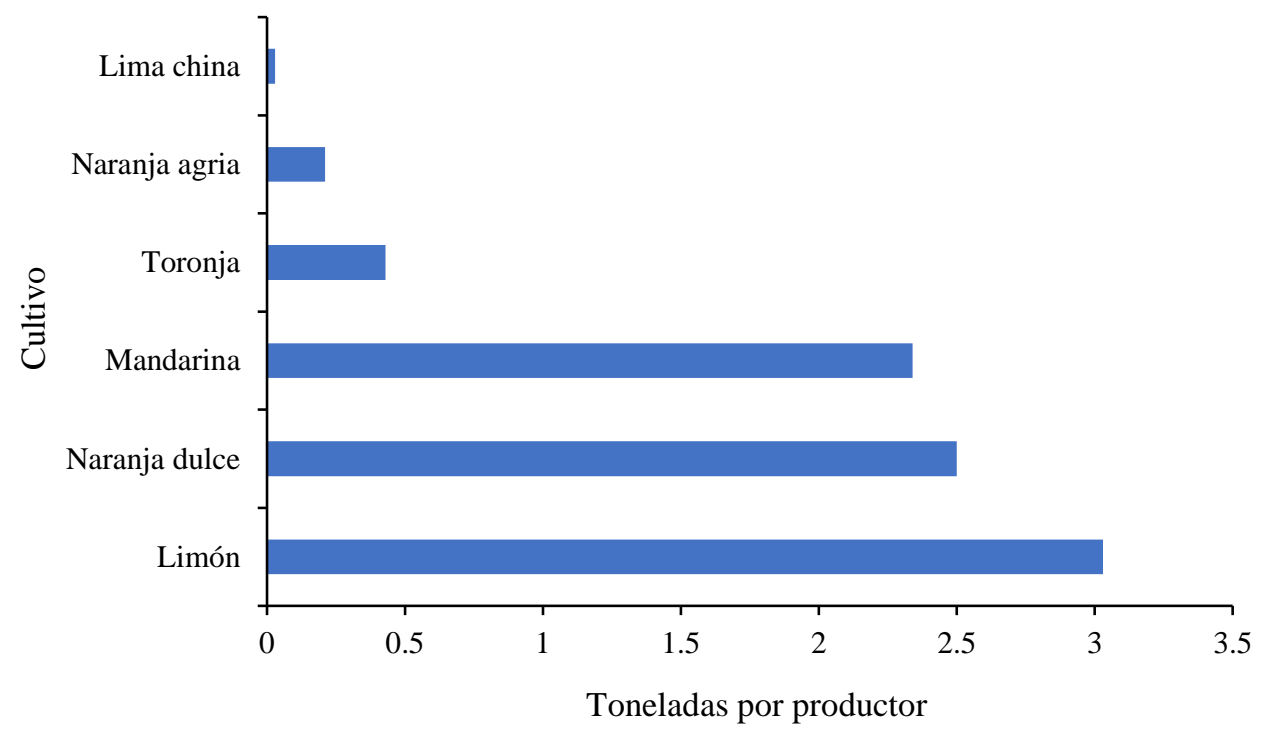

Figura 2. Rendimiento de la producción de cítricos en el Estado de Campeche.

Está por debajo del reportado para el estado de Campeche. Con una producción de $7.539 \mathrm{t} \mathrm{ha}^{-1}$ (SIAP, 2017) y está muy lejos de alcanzar los niveles de producción de grandes estados productores como Michoacán, quien ocupó el primer lugar en producción de limón con un rendimiento de 16.047 t/ha (Maya, 2017; Orduz-Rodríguez et al., 2017); este bajo rendimiento puede explicarse a que la población encuestada se dedica a la citricultura sin tecnificar, además de las condiciones climáticas contrastantes presentes en la actualidad en estas regiones.

De manera que, la vulnerabilidad que se tiene a inundación por mareas de tormenta, el aumento de las lluvias en algunas zonas y la disminución de ellas, así como el aumento de temperatura genera pérdida y modificación de la biodiversidad en municipios del Carmen, Champotón, Campeche, Tenabo, Hecelchakan y Calkini. Los asentamientos humanos e infraestructuras estratégicas ubicados en zona de costa son los más expuestos y sensibles a los eventos climáticos como los ciclones, frentes fríos y nortes.

Las consecuencias de estos eventos crecen con la subida del nivel del mar y las mareas de tormenta que tienen como consecuencia inundaciones costeras y penetración del agua de mar (Gobierno del Estado de Campeche, 2015). Por otra parte, no existe correlación entre el tamaño total del ejido o propiedad con el área destinada al cultivo, de acuerdo con la correlación de Pearson $(r=0.32)$. Se reportan un total de siete tipos de cítricos cultivados, un solo propietario puede tener hasta cinco cultivos de cítricos.

Sin embargo, la diversificación de cultivos tampoco está correlacionada con el tamaño de la propiedad $(\mathrm{r}=0.032)$, ni con el área destinada al cultivo de cítricos $(\mathrm{r}=0.33)$. Así mismo, no se encontró correlación con la producción total y el tamaño del área de cultivo de cítricos $(\mathrm{r}=0.47)$. 
Por tanto, se percibe que las decisiones del productor, sobre qué tipo de cultivos establecer y la tendencia del uso de la tierra para la citricultura, depende únicamente de la decisión personal del productor, el cual no tiene acceso a servicios de extensionismo y o asesoría técnica especializada. Así como a la facilidad que tenga para comercializar sus productos. En este sentido, AlmaguerVargas y Ayala-Garay (2014) mencionan que en México, el incremento de la producción de limón ha estado basado en un aumento en la superficie y no en innovaciones que mejoren el rendimiento, debido a que la transferencia y la adopción de innovaciones han tenido múltiples limitantes. Así como por la reducida cobertura de la asistencia técnica pública de menos del $1 \%$ de las unidades productivas en el país y el uso de metodologías ineficientes para ello (Almaguer-Vargas y AyalaGaray, 2014; Lucatello, 2014). Donde la percepción adecuada es un requisito previo a la adaptación exitosa de las estrategias agrícolas (Makuvaro et al., 2018).

\section{Conocimiento sobre el cambio climático}

Los resultados en este estudio reflejan que el $66.6 \%$ de las personas entrevistadas declara haber escuchado sobre el CC, de los cuales el $4.5 \%$ no se encuentra realmente informado sobre el tema, $86.3 \%$ está poco informado y $9 \%$ se considera bastante informado sobre la temática de CC. Guerrero-Carrera et al. (2015) reportaron en agroecosistemas de caña de azúcar de Veracruz, México, que el $81 \%$ de los agricultores habían oído hablar del CC, el 97\% percibió cambios en el clima, de ellos, el 73.5\% percibió cambios en temperatura, 87.8\% percibió cambios en la precipitación, $49 \%$ en el viento y $69 \%$ reclamaron daños a sus agroecosistemas por el CC.

Esto significa que muchos de los productores cuentan con la información referente a este tema y han percibido sus efectos. Evans y Durant (1995) mencionan que el nivel de conocimiento condiciona la calidad de la percepción; en este sentido, no parece que el nivel de conocimiento determine la percepción individual, si bien, por el contrario, la estabiliza y consolida, ya sea positiva o negativamente. Además, las fuentes más relevantes donde los productores de cítricos han obtenido información sobre CC son la televisión, seguido por los técnicos y por el periódico, las otras fuentes incluidas en este estudio no tienen relevancia por su bajo impacto (Figura 3 ).

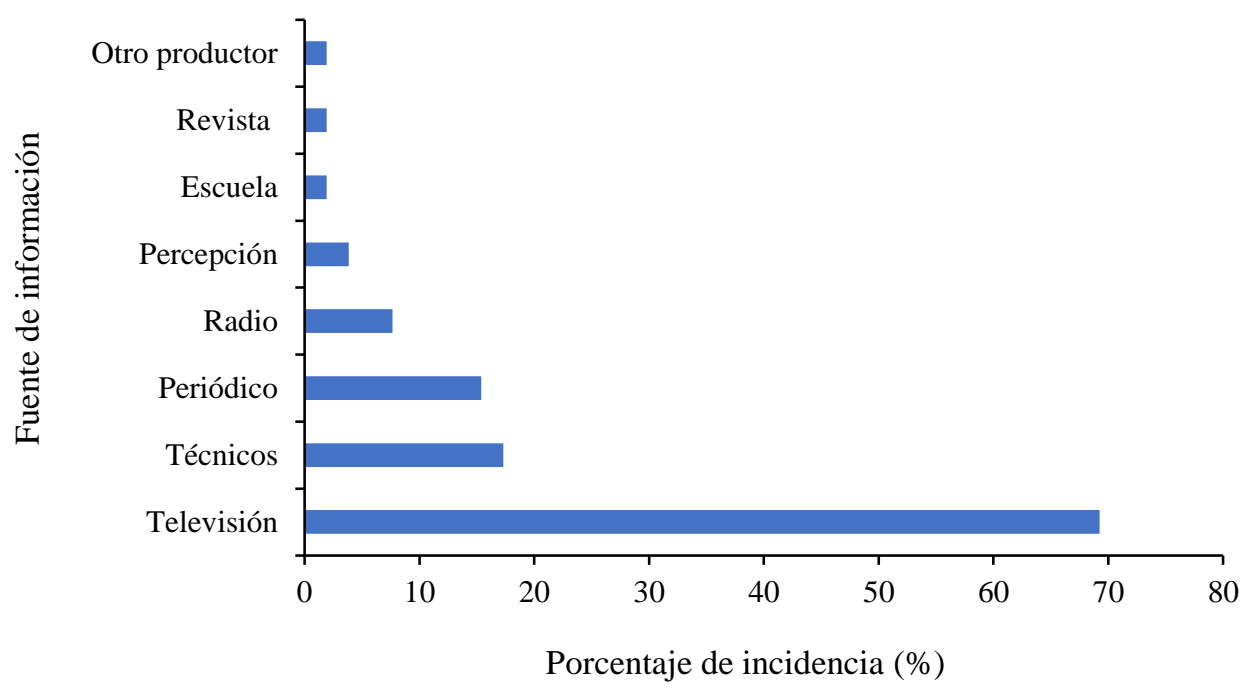

Figura 3. Medios informativos y de difusión sobre cambio climático. 


\section{Percepción sobre el efecto del cambio climático en la agricultura}

Cuando se cuestionó sobre la percepción del cambio climático, el 1.5\% considera no percibir un cambio, $28.8 \%$ ha percibido un poco de cambio y $69.6 \%$ percibe un cambio mayor. El $43 \%$ cree que el clima se modifica por causas humanas, $27.6 \%$ atribuye a causas naturales; no obstante, el $24.6 \%$ considera que estos dos factores influyen en el CC. Entonces, esto indica que el CC es percibido por la mayoría de los productores de cítricos; también Zhang et al. (2017); Karimi et al. (2018) coinciden con estas opiniones sobre la percepción de CC y los factores que aceleran el calentamiento global; aunado a ello, Márquez (2016) menciona que el CC si bien, es percibido, su apreciación no es tan clara, tal como lo demostraron funcionarios municipales de Campeche, México, en donde su percepción y conocimiento estaban basados en la televisión y en el internet. Por otra parte, el análisis de componentes principales muestra una varianza del $63.5 \%$ en los dos primeros componentes obtenidos para esta variable (Cuadro 1).

Cuadro 1. Análisis de componentes principales para la percepción de los factores ambientales en Campeche.

\begin{tabular}{ccc}
\hline Eventos climáticos & Componente 1 & Componente 2 \\
\hline Sequías prolongadas & & $0.695^{* *}$ \\
Fuertes lluvias & -0.49 & -0.343 \\
Huracanes & $-0.533^{*}$ & 0.223 \\
Inundaciones & $-0.597^{*}$ & -0.223 \\
Vientos fuertes & -0.345 & $0.548^{* *}$ \\
\hline
\end{tabular}

${ }^{*}=$ no existe cambio; ${ }^{* *}=$ si existe cambio.

El primer componente está relacionado con los fenómenos hídricos (los huracanes y las inundaciones) y tiene la mayor relevancia en la percepción del CC, y el segundo componente se asocia con las sequías y los vientos. Un grupo de personas pertenecientes al primer componente opina que los huracanes y las inundaciones no tienen un impacto importante sobre el cultivo de cítricos. Un segundo grupo de personas opina verse afectado por sequías y vientos (Figura 4).

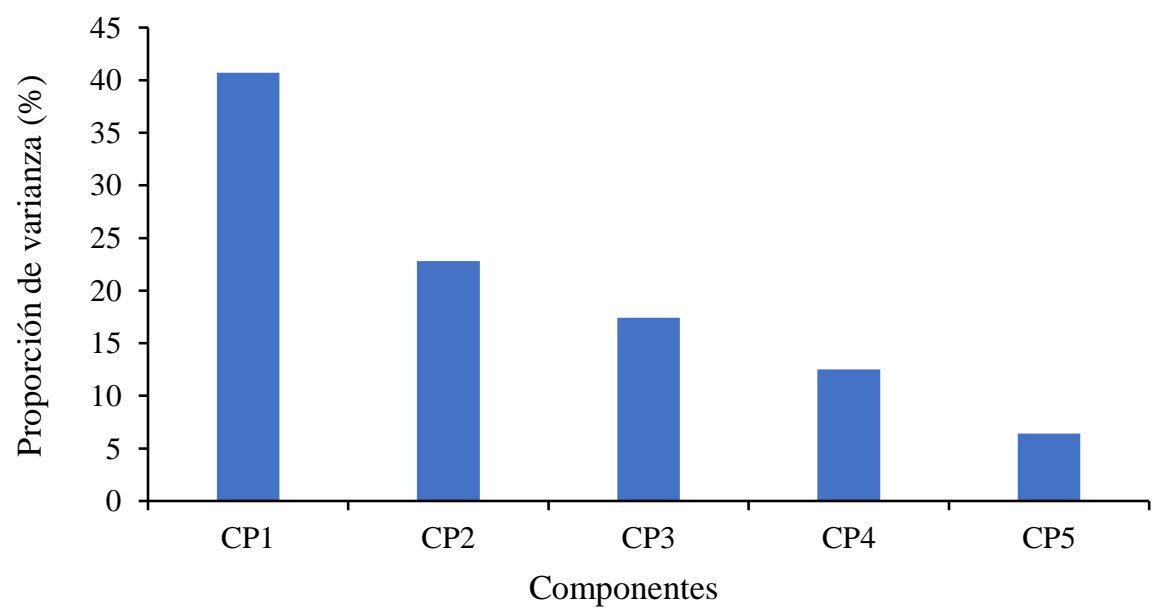

Figura 4. Análisis de componentes principales sobre la percepción del cambio climático en citricultores de Campeche. 


\section{Efectos del cambio climático en el cultivo de cítricos}

Los huracanes ocasionan pérdida total de árboles, además de presencia de enfermedades y plagas por exceso de humedad por las lluvias fuertes. El grado de afectación que los productores del estado de Campeche reportaron por desastres naturales en su sistema de producción citrícola fue de la siguiente manera: a $18.5 \%$ les ha afectado mucho, a $70.3 \%$ ha afectado poco y a $11.1 \%$, nada.

Además, los productores mencionan que no sólo es un desastre el que afecta a sus cítricos, sino que ellos detectan a varios fenómenos ocurridos durante las diferentes temporadas del año, reportando a las sequías prolongadas como las de mayor frecuencia. Así mismo, el 54.5\% ha detectado una disminución en su producción después de un desastre, aunque no tienen reportes exactos de dichas pérdidas.

Campeche, así como el Distrito Federal, Baja California, Baja California Sur y Durango son dos veces menos vulnerables que Chiapas, Veracruz, Tamaulipas, Oaxaca y Sinaloa para el caso de inundaciones y eventos relacionados, entre los factores determinantes de ello se encuentra el uso de suelo que integra indicadores de las actividades antropogénicas y su efecto sobre la reducción de las áreas naturales, por lo que la vulnerabilidad de Campeche se debe a su tipo de relieve (cárstico). La degradación del suelo es otro elemento importante, de acuerdo con un análisis realizado, debido a las inundaciones, sequías y granizadas (Ibarrarán et al., 2014).

Aunque los rezagos en la capacidad económica y social también pueden incidir en la vulnerabilidad ante desastres y la resiliencia frente a ellos (Ibarrarán et al., 2014). De esta manera, los efectos de sequías prolongadas, de lluvias fuertes, de huracanes y de vientos fuertes son relacionados con la pérdida de ramas, hojas, flor y frutos en árboles de cítricos. Así mismo, en la Península de Yucatán la sequía produce pérdidas y bajo rendimiento de cultivos, de cabezas de ganado y de colmenas, disminución de la calidad de los productos, así como incremento en los costos de producción, lo que produce reducción en el ingreso de los productores (Estrada-Medina et al., 2016).

De la misma manera, Mora-Aguilera et al. (2016) Encontraron que el incremento en la frecuencia de las lluvias ha afectado eventos biológicos de las plagas, por lo que la relación entre plagas exóticas detectadas históricamente y el número de huracanes ocurridos en México es altamente significativa $\left(r^{2}=0.98\right)$; entre estas plagas específicas, relacionadas con los huracanes se pueden citar a los Trips Palmi y a la cochinilla Rosada.

\section{Prácticas tecnológicas ante el efecto del cambio climático}

En esta investigación no se registró un cambio significativo en el uso de prácticas tecnológicas después de un desastre natural con excepción de la labranza, de la cual incrementan su práctica (McNemar.test $\left.\chi^{2}=10.56, p<0.01^{* *}\right)$ (Cuadro 2).

No obstante, los productores de cítricos si realizan otras prácticas de tipo agroecológicas para reducir la vulnerabilidad ante inundaciones como: realizar desagües y hacer canales; ante sequía: tener sistema de riego, pozos, cuidar el agua y dejar rastrojo en el suelo para mantener la humedad, ante vientos: sembrar árboles que sirven como cortinas rompe vientos y colocar soportes a las plantas. 
Cuadro 2. Prueba de McNemar para detectar cambios en el uso de tecnologías después de un evento climático.

\begin{tabular}{ccc}
\hline Tecnología & Chi-cuadrada & $p$ \\
\hline Labranza & 10.56 & $<0.01^{* *}$ \\
Control de malezas & 2.25 & $0.133^{\mathrm{ns}}$ \\
Fertilización & 0.25 & $0.617^{\mathrm{ns}}$ \\
Plagas & 0.57 & $0.449^{\mathrm{ns}}$ \\
Riego & 0 & $1^{\mathrm{ns}}$ \\
Poda & 1.45 & $0.227^{\mathrm{ns}}$ \\
\hline
\end{tabular}

${ }^{* *}=$ cambio en el uso de tecnología por evento climático.

Los efectos del CC pueden reducir la eficiencia y eficacia al implementar las prácticas tecnológicas de conservación agrícolas y afectar la capacidad de los agricultores para desarrollarse (Wagena and Easton, 2018), además de que aumentan la volatilidad de los precios de los alimentos (LópezFeldman y Hernández-Cortés, 2016), convirtiéndose en una amenaza potencial para la seguridad y soberanía alimentaria de los países en desarrollo en formas complejas (Kusangaya et al., 2014; Golcher et al., 2017).

Por otro lado, hace falta diseñar estrategias de mayor difusión y promover prácticas una vez que el citricultor tenga evidencias de afectaciones por este fenómeno e incluso antes de ellas. Ante este escenario, Núñez et al. (2017) proponen algunas estrategias como: la selección de portainjerto como elemento clave y la inoculación con cepas adecuadas de hongos micorrízicos arbusculares, para contribuir a la tolerancia tanto al estrés hídrico como salino, y a la aplicación de algunos agentes químicos.

De la misma manera, algunas prácticas agroecológicas para combatir la erosión reportadas por Debray et al. (2019) son los agujeros de zai (pozos excavados en la pretemporada para atrapar agua), medias lunas, fardos de piedra y terrazas para rehabilitar las tierras de cultivo, evitar la pérdida de suelo por la erosión del agua, y reducir la erosión eólica.

\section{Conclusiones}

El efecto principal del CC en los agroecosistemas de cítricos con base en la percepción de sus manejadores, es que sus rendimientos están decreciendo. Un hecho que se agravará en el mediano y largo plazo con base en los escenarios propuestos que indican un aumento de la temperatura y una reducción de la precipitación. Ante esta situación, es fundamental que el productor diseñe e implemente estrategias encaminadas a un mejor manejo de la fertilidad y humedad del suelo, a la conservación de la diversidad y agrobiodiversidad asociada a este agroecosistema.

Al no hacerlo se generarían condiciones de posibilidad para que este tipo de agroecosistema desaparezca progresivamente y con ello, su función como abastecedor de una fruta importante para la alimentación de la población. Es fundamental señalar que, aunque los productores de cítricos perciben los efectos del CC en sus agroecosistemas de cítricos y la mayoría de ellos indica haber escuchado hablar sobre este fenómeno, éstos aún no cuentan con los conocimientos necesarios para el diseño de estrategias sobre cómo enfrentar sus efectos. 
Esto se debe fundamentalmente a la complejidad del fenómeno y a la forma en que ha sido comunicado, así como a su relación con fenómenos cada día más recurrentes y severos como las lluvias torrenciales, huracanes y vientos fuertes, elementos agroclimáticos relacionados con la pérdida de ramas, hojas, flores y frutos que ocasionan pérdida en la cantidad y calidad del producto, lo cual limita su comercio en mercados nacionales o internacionales para la obtención de precios justos que les permitan cubrir las necesidades básicas de ellos y de sus familias.

Entre éstas y otras acciones deberán ser fomentadas y fortalecidas por políticas públicas como estrategias potenciales para combatir y contrarrestar los efectos del CC. De este modo se apoyará al sector citrícola y con ello, a las familias cuya economía se basa en los ingresos generados por la citricultura, además de contribuir a la seguridad y soberanía alimentaria de la región y del país.

\section{Literatura citada}

Agovino, M.; Casaccia, M.; Ciommi, M.; Ferrara, M. and Marchesano, K. 2018. Agriculture, climate change and sustainability: The case of EU-28. Ecological Indicators. https://doi.org/10.1016/j.ecolind.2018.04.064.

Almaguer-Vargas, G. y Ayala-Garay, A. V. 2014. Adopción de innovaciones en limón 'Persa' (Citrus latifolia tan.) en Tlapacoyan, Veracruz. Uso de bitácora. Revista Chapingo Ser. Hortic. 20(1):89-100.

Bahena-Delgado, G. y Tornero-Campante, M. A. 2009. Diagnóstico de las unidades de producción familiar en pequeña irrigación en la subcuenca del río Yautepec, Morelos. Economía, Sociedad y Territorio. 9(29):165-184.

Camargo-López, J. y Espericueta-Reyna, T. 2006. La problemática de la citricultura campesina del barretal Tamaulipas. Sociedades Rurales, Producción y Medio Ambiente. 6(13): 31-50.

Corona, J. M. A. 2018. El conocimiento, la percepción y disponibilidad para afrontar el cambio climático en una población emergente, los migrantes de retorno. Estudios Sociales. 28(52):1-28.

Debray, V.; Wezel, A.; Lambert-Derkimba, A.; Roesch, K.; Lieblein, G. y Andrew, F. C. 2019. Agroecological practices for climate change adaptation in semiarid and subhumid Africa. Agroecology and Sustainable Food Systems. 43(4):429-456.

Dussel-Peters, E. 2002. Territorio y competitividad en la agroindustria en México: condiciones y propuestas de política para los clusters del limón mexicano en Colima y la piña en Veracruz. Ed. Plaza y Valdez. México, DF. 173 p.

ECOSUR (El Colegio de la Frontera Sur). 2012. Unidad Campeche. Inventario Estatal de Emisiones de Gases de Efecto Invernadero en el Estado de Campeche.

Evans, G. y Durant, J. 1995. The Relationship between Knowledge and Attitudes in Public Understan-ding of Science in Britain. Public Understanding of Science. 4(1): 57-74.

Estrada-Medina, H.; Cobos-Gasca, V.; Acosta-Rodríguez, J. L.; Peña-Fierro, S.; Castilla-Martínez, M.; Castillo-Carrillo, C.; Franco-Brito, S.; López-Castillo, D.; López-Díaz, M.; LunaFlores, W.; Maldonado-Repetto, A.; Álvarez-Rivera, O.; Cámara-Romero, J. L.; MoralesGuadarrama, A.; Moreno-Arjona, A. M.; Pérez-Niño, B.; Rodríguez-Lara, P. y ZapataLuna, R. L. (2016). La sequía de la península de Yucatán. Tecnología y Ciencias del Agua. 7(5):151-165. 
Galindo, G., G.; Tabares, R. W. C. y Gómez, A. G. 2000. Caracterización de productores agrícolas de seis distritos de desarrollo rural de Zacatecas. Terra Latinoamericana. 18(1):83-92.

Gobierno del estado de Campeche. 2015. Programa estatal de acción ante el cambio climático, Visión 2015-2030. Secretaría de Medio Ambiente y Aprovechamiento Sustentables. Campeche, México. 113 p.

Golcher, C. S.; Arts, B. and Visseren-Hamakers, I. 2017. Seeing the forest, missing the field: Forests and agriculture in global climate change policy. Land Use Policy. 77:627-640.

Guerrero-Carrera, J.; Landeros-Sánchez, C.; Martínez-Dávila, J. P.; López-Romero, G.; NikolskiiGavrilov, I.; Hernández-Pérez, J. M. and Soto-Estrada, A. 2015. Climate change impacto in sugarcane crop in the Gulf of México: A Farmer perception and adaptation Measures. Journal of Agriculture of Science. 7(10):140-158.

Hernández-Sampieri. R.; Fernández-Collado, C. y Baptista-Lucio, P. 1991. Metodología de la Investigación. Mac Graw Hill. México. 850 p.

Ibarrarán, M. E.; Reyes, M. y Altamirano, A. 2014. Adaptación al cambio climático como elemento de combate a la pobreza. Región y Sociedad. XXVI(61):5-50.

INEGI (Instituto Nacional de Geografía y Estadística). 2012. Perspectiva estadística de Campeche. Instituto Nacional de Estadística y Geografía.

IPCC. (Grupo Intergubernamental de Expertos sobre el Cambio Climático). Cambio climático. 2007: Informe de síntesis. Ginebra: 2007.

Islam, M. T. and Nursey-Bray, M. 2017. Adaptation to climate change in agriculture in Bangladesh: The role of formal institutions. Journal of Environmental Management. 200:347-358.

Karimi, V.; Karami, E. and Keshavarz, M. 2018. Climate change and agriculture: Impacts and adaptive responses in Iran. Journal of Integrative Agriculture. 17(1):1-15.

Klocker, N.; Head, L.; Dun, O. and Spaven, T. 2018. Experimenting with agricultural diversity: Migrant knowledge as a resource for climate change adaptation. Journal of Rural Studies. 57:13-24.

Kusangaya, S.; Warburton, M. L.; Van Garderen, E. A. and Jewitt, G. P. 2014. Impacts of climate change on water resources in southern Africa: A review. Physics and Chemistry of the Earth, Parts A/B/C. 67:47-54.

López-Feldman, A. J. y Hernández-Cortés, D. 2016. Cambio climático y agricultura: una revisión de la literatura con énfasis en América Latina. El trimestre económico. 83(332):459-496.

Lucatello, S. 2014. La Cooperación Internacional y medio ambiente: tendencias y desafíos para Latinoamérica. Revista Internacional de Cooperación y Desarrollo. 1(2):33-56.

Makate, C.; Makate, M.; Mango, N. and Siziba, S. 2019. Increasing resilience of smallholder farmers to climate change through multiple adoption of proven climate-smart agriculture innovations. Lessons from Southern Africa. Journal of Environmental Management. 231:858-868.

Makuvaro, V.; Walker, S.; Masere, T. P. and Dimes, J. 2018. Smallholder farmer perceived effects of climate change on agricultural productivity and adaptation strategies. Journal of Arid Environments. 152:75-82.

Márquez, R. I. 2016. Conocimientos y percepciones sobre el cambio climático en personal de la administración pública municipal del estado de Campeche. Revista Iberoamericana de Producción Académica y Gestión Educativa. 3(5):1-8.

Maya, A. C. J. 2017. Cítricos mexicanos en el mercado japonés: experiencias y oportunidades para Sinaloa. México y la Cuenca del Pacífico. 6(16):107-142. 
Mora-Aguilera, G.; Acevedo-Sánchez, G.; Calderón-Estrada, G.; Flores-Sánchez, J.; DomínguezMonge, S.; Peter, B. y González-Gómez, R. 2016. Consideraciones epidemiológicas del cambio climático en la fitosanidad de cultivos tropicales. Revista Mexicana de Fitopatología. 32(2):147-167.

Morett-Sánchez, J. C. y Cosío-Ruiz, C. 2017. Panorama de los ejidos y comunidades agrarias en México. Agricultura Sociedad y Desarrollo. 14(1):125-152.

Núñez, V. M.; Dell' Amico, R. J.; Pérez, H. M. C. y Betancourt, G. M. 2017. Estrés hídrico y salino en cítricos. Estrategias para la reducción de daños. Cultivos Tropicales. 38(4):65-74.

Orduz-Rodríguez, J. O.; Garzón, C. y Lucia, D. 2017. Alternancia de la producción y comportamiento fenológico de la naranja 'Valencia' (Citrus sinensis [L.] Osbeck) en el trópico bajo húmedo de Colombia. Revista Corpoica-Ciencia y Tecnología Agropecuaria. 13(2):136-144.

Pérez, G.; Vilches, A.; Grimaldi, J. C. T. y Álvarez, O. M. 2006. Década de la educación para un futuro sostenible (2005-2014): un punto de inflexión necesario en la atención a la situación del planeta. Revista Iberoamericana de Educación. Madrid. 125-178 pp.

Rosales, M. V.; Martínez, D. J. P. y Galicia, G. F. 2015. Cambios en la estructura y el funcionamiento de agroecosistemas por migración familiar en Jamapa, Veracruz, México. Rev. Mex. Cienc. Agríc. 6(1):59-70.

Rosales-Martínez, V. y Leyva-Trinidad, D. A. 2019. El rol de la mujer en el agroecosistema y su aporte a la producción de alimentos. Agroproductividad. 12(1):47-52.

SAGARPA (Secretaría de Agricultura, Ganadería, Desarrollo Rural, Pesca y Alimentación). 2018. Atlas agroalimentario 2012-2018. https://nube.siap.gob.mx/gobmx_publicaciones_ siap/pag/2018/Atlas-Agroalimentario-2018.

Sandoval, C. y Soares-Munguía, M. T. 2014. Vulnerabilidad social y percepciones asociadas al cambio climático: Una aproximación desde la localidad de Ixil, Yucatán. Sociedad y Ambiente. 1(5):7-24.

SIAP (Servicio de Información Agroalimentaria y Pesquera). 2019. Producción agrícola. México. Sagarpa. https://www.gob.mx/siap/acciones-y-programas/produccion-agricola-33119.

SIAP (Servicio de Información Agroalimentaria y Pesquera). 2017. Avance de Siembras y Cosechas Resumen nacional por estado. https://www.gob.mx/siap.

Sierra, B. R. 1995. Técnicas de investigación social. Teoría y ejercicios. Ediciones Paraninfo. Madrid, España. 720 p. ISBN: 9788428324298.

Thornton, P. K.; Whitbread, A.; Baedeker, T.; Cairns, J.; Claessens, L.; Baethgen, W.; Bunn, C.; Friedmann, M.; Giller, K. E. y Herrero, M. 2018. A framework for priority-setting in climate smart agriculture research. Agricultural Systems. 167:161-175.

TRIBUNA, 2020. Productora de yemas de cítricos en Campeche. Informativa Campeche, 200601-16. www.sicde.gob.mx/portal/bin/nota.php?from=60\&accion=buscar\&subrutina= pagina_1 \& column $=2 \&$ busqueda $=\&$ orderBy=Notas.MedioComunicacion $\&$ order $=$ ASC\& notaId $=6601419743 \mathrm{cbb} 9 \mathrm{~b} 0 \mathrm{be} 9 \mathrm{cb}$.

Wagena, B. M. and Easton, M. Z. 2018. Agricultural conservation practices can help mitigate the impact of climate change. Science of the Total Environment. 635:132-143.

Zhang, P.; Zhang, J. and Chen, M. 2017. Economic impacts of climate change on agriculture: The importance of additional climatic variables other than temperature and precipitation. Journal of Environmental Economics and Management. 83:8-31. 